\title{
Photon Statistics in the Stochastic Limit of QED without Dipole Approximation ${ }^{1}$
}

\author{
L. Accardi and Y. G. Lu \\ Centro Vito Volterra, Universitè di Roma Tor Vergata, 00133 Rome, Italy \\ Dipartimento di Matematica, Universitè di Bari, Italy \\ e-mail: accardi@volterra.uniroma2.it; lu@dm.uniba.it \\ Received October 12, 2006
}

\begin{abstract}
In this paper we investigate the distribution of the Heisenberg evolution of the number operator in the stochastic limit of the quantum electromagnetic field without dipole approximation. We prove that in this case the usual Poisson statistics of the free fields has to be replaced by a hypergeometric series whose coefficients we compute explicitly. The physical effects of the new statistics should manifest themselves in situation which are not yet in the relativistic regime, but in which the dipole approximation is no longer valid.
\end{abstract}

PACS numbers: 42.50.Ar

DOI: $10.1134 / \mathrm{S} 0030400 \mathrm{X} 07080176$

\section{INTRODUCTION}

It is known (see [1]) that, in the stochastic limit of strongly nonlinear interactions dramatically new physical and mathematical features arise, for example, the breaking of the usual commutation relations and their replacement by new "entangled commutation relations," which are responsible for richer and more interesting statistics, the replacement of usual Fock by "interacting" Fock space, and the emergence of Hilbert modules rather than Hilbert spaces as natural quantum state spaces.

In the present paper this class of phenomena will be illustrated with the case of the stochastic limit of QED without dipole approximation. More precisely we prove that, in this limit, the photon statistics, although more complex, can still be explicitly calculated and is expressed by a series whose coefficient can be split into a product of two terms, one that is a matrix element of the atomic part and a combinatorial one that is given by a hypergeometric series (see [2]).

In Section 4 (see formula (1)) we introduce the main object studied in the present paper, i.e., the vacuum mean value, denoted $V\left(t, T_{G}\right)$, of any function $G$ of the time- $t$-evolved number operator, i.e., the photon statistics at time $t$. Our main result is condensed in formula (9) of Section 5, which expresses this distribution as a hypergeometric series whose coefficients have a simple expression given in terms of the atomic momentum $p$ and the generalized (moment dependent) susceptivities $\left(F_{g} \mid F_{g}\right)_{-}$(see formulas $\left.(4),(5)\right)$.

\footnotetext{
${ }^{1}$ The text was submitted by the authors in English.
}

\subsection{Notations}

For a nonrelativistic particle with mass $m$ and charge $e$ interacting with the quantum electromagnetic (QEM) field with vector potential $\mathbf{A}$, the total Hamiltonian $H$ has the form

$$
H=H_{S}+H_{R}+H_{I},
$$

where: (i) $H_{S}$ is the free Hamiltonian of the particle

$$
H_{S}:=-\frac{\hbar^{2}}{2 m} \Delta+V(\mathbf{x})
$$

(ii) $H_{R}$ is the free Hamiltonian of the QEM field

$$
H_{R}:=\sum_{k} \hbar \omega(k) a_{k}^{+} a_{k},
$$

(iii) $H_{I}$ is the interaction Hamiltonian between the particle and the QEM field which has the form

$$
\frac{i e \hbar}{m} \mathbf{A} \cdot \nabla+\frac{e^{2}}{2 m} \mathbf{A} \cdot \mathbf{A}
$$

and if we assume that the QEM field considered is not very strong, then the $A \cdot A$-term in the interaction Hamiltonian can be neglected i.e.,

$$
H_{I}:=\frac{i e \hbar}{m} \mathbf{A} \cdot \nabla
$$

We have proved elsewhere that effectively, in the stochastic limit, the $A \cdot A$-term tends to zero. 
It is well known that, for the Hamiltonian $H_{S}$, one has two typical choices:

(a) $H_{S}$ has a discrete spectrum, e.g., $H_{S}=$ $\sum_{j} E_{j} b_{j}^{+} b_{j}$, where $b_{j}^{+}, b_{j}$ are independent Fermi creation-annihilation operators, $E_{j}$ are positive (the simplest case is that of a two-level particle for which $H_{S}=$ $\left.E_{0} b_{0}^{+} b_{0}+E_{1} b_{1}^{+} b_{1}\right)$.

(b) The particle has a continuous energy spectrum.

In the present paper we assume that scalar potential $V$ is so small that it can be neglected. Thus the "free" particle Hamiltonian is the Laplacian on full space

$$
H_{S}=-\frac{\hbar^{2}}{2 m} \Delta
$$

(therefore, the energy spectrum is absolutely continuous).

The interaction Hamiltonian can be put in the form

$$
\begin{gathered}
\frac{e}{m} \sum_{k, \sigma} \frac{u_{h}}{\sqrt{\omega_{k}} \mathbf{A} \cdot \mathbf{p}} \\
=\frac{e}{m} \sum_{k, \sigma} \frac{u_{h}}{\sqrt{\omega_{k}}} \mathbf{p}_{\sigma}\left(a_{k, \sigma}^{+} e^{-i \mathbf{k} \cdot \mathbf{q}}+a_{k, \sigma} e^{i \mathbf{k} \cdot \mathbf{q}}\right),
\end{gathered}
$$

where $\mathbf{p}, \mathbf{q}$ are the momentum, position operator of the particle and $u_{h}$ is a constant depending on the velocity of light and the Planck constant $\hbar$.

In order to obtain useful information on the QEM field, some approximations are usually introduced to simplify the problem. Among them, the most widely used is the so-called dipole approximation, which replaces the exponential factor $e^{ \pm \mathbf{i k} \cdot \mathbf{q}}$ in (6) by 1 .

This approximation is reasonable when the wave length of the QEM field is larger than the Bohr radius of the particle. But it is inadequate for super-short impulses and also for multi-mode photons. Therefore, an important problem is to study the interaction of the QEM fields with a particle outside the dipole approximation. This problem was first investigated in $[3,4]$ and in this paper we study again the case (b).

For notational simplicity we neglect the explicit form of all constants appearing in Hamiltonians and write

$$
\begin{gathered}
H_{S}:=-\Delta / 2, \\
H_{R}:=\sum_{k} \omega(k) a_{k}^{+} a_{k} \approx \int_{\mathbf{R}^{d}} \omega(k) a_{k}^{+} a_{k} d k,
\end{gathered}
$$

$$
\begin{gathered}
H_{S}=\lambda \sum_{k, \sigma} p_{\sigma}\left(a_{k, \sigma}^{+} e^{-i \mathbf{k} \cdot \mathbf{q}} g_{0}(k)+a_{k, \sigma} e^{i \mathbf{k} \cdot \mathbf{q}} \bar{g}_{0}(k)\right) \\
\approx \lambda \int_{\mathbf{R}^{d} \sigma} \sum_{\mathbf{p}}\left(a_{k, \sigma}^{+} e^{-i \mathbf{k} \cdot \mathbf{q}} g_{0}(k)+a_{k, \sigma} e^{i \mathbf{k} \cdot \mathbf{q}} \bar{g}_{0}(k)\right),
\end{gathered}
$$

where $\lambda$ is the coupling constant; $g_{0}$ is a Schwartz function which comes from the combination of the dispersion function $1 / \sqrt{\omega(k)}$ with a cutoff; the polarization index $\sigma$ runs over a two elements set, e.g., $\{0,1\}$. Moreover, the usual boson commutation relations are assumed:

$$
\left[a_{k, \sigma}, a_{k^{\prime}, \sigma^{\prime}}^{+}\right]=\delta_{\sigma, \sigma^{\prime}} \delta\left(k-k^{\prime}\right) .
$$

In the following investigation, we shall also neglect the polarization index $\{0,1\}$.

\subsection{Previous Results on Stochastic Limit of QED}

In this section as a way to introduce our notations, we quickly summarize our previous results on the stochastic limit of QED and we refer to the book [1] for a systematic exposition and more details.

The evolution operator in the interacting picture is

$$
U_{t}^{(\lambda)}:=e^{i t\left(H_{S}+H_{R}\right)} e^{-i t\left(H_{S}+H_{R}+\lambda H_{I}\right)} .
$$

In QED the quantity $e / m$, i.e., the coupling constant $\lambda$ is small. The basic idea of the stochastic limit is to single out the asymptotic behavior of the relevant dynamical quantities when the time $t$ tends to infinity by the rescaling $t$ and the coupling constant $\lambda$ to zero. This is equivalent to replace the time parameter $t$ by $t / \lambda^{2}$.

It was proved in $[3,4]$ that, without assuming the dipole approximation, the limit

$$
\lim _{\lambda \rightarrow 0} U_{t / \lambda^{2}}^{(\lambda)}=: U(t)
$$

exists and satisfies a new type of quantum stochastic differential equation of the form

$$
\begin{aligned}
U(t)= & 1+\int_{0}^{t}\left(d A_{s}^{+}\left(F_{g}\right)(-i \mathbf{p})-(i \mathbf{p})^{+} d A_{s}\left(F_{g}\right)\right. \\
& \left.-(i \mathbf{p})^{+}\left(F_{g} \mid F_{g}\right)_{-}(i \mathbf{p}) d s\right) U(s),
\end{aligned}
$$

where

(i) all quantum stochastic processes live on the QED Hilbert module $\Gamma$, over the momentum algebra $\mathscr{P}$ of the atom,

(ii) $\mathscr{P}$ is the momentum $W^{*}$-algebra $L^{\infty}(\mathbf{p})$ generated by $\left\{e^{i \mathbf{p} \cdot \mathbf{k}} ; k \in \mathbf{R}^{d}\right\}$, 
(iii) $\mathscr{F}$ is defined as the $\mathscr{P}$-linear module generated by the set,

$$
\begin{gathered}
\left\{F_{f}(t):=\int_{\mathbf{R}^{d}}\left(S_{t} f\right)(k) e^{-i k \mathbf{q}} e^{i k \mathbf{p}} d k,\right. \\
\left.f \text { is Schwartz function on } \mathbf{R}^{d}\right\}, \\
S_{t} f(k):=e^{-i t \omega(k)} f(k),
\end{gathered}
$$

(iv) for each $n \in \mathbf{N}$, the module inner (not scalar) product on $\left(L^{2}\left(\mathbf{R}^{d}\right) \otimes \mathscr{F}\right)^{\otimes n}$, as a right module on $L^{\infty}(\mathbf{p})$, is defined as

$$
\begin{gathered}
\left(\left(\alpha_{1} \otimes F_{f_{1}}\right) \otimes\left(\alpha_{2} \otimes F_{f_{2}}\right)\right. \\
\otimes \ldots\left(\alpha_{n} \otimes F_{f_{n}}\right) \mid\left(\beta_{1} \otimes F_{g_{1}}\right) \otimes\left(\beta_{2} \otimes F_{g_{2}}\right) \\
\left.\otimes \ldots\left(\beta_{n} \otimes F_{g_{n}}\right)\right):=\prod_{h=1}^{n}\left\langle\alpha_{h}, \beta_{h}\right\rangle_{L^{2}\left(\mathbf{R}^{d}\right)} \\
\times \int_{\mathbf{R}^{d}} d u_{1} \ldots d u_{n} \int_{\mathbf{R}^{d}} d k_{1} \ldots d k_{n} \\
\times \prod_{h=1}^{n}\left(e^{i u_{h} k_{h} \mathbf{p}} \bar{f}_{h}\left(k_{h}\right)\left(S_{u_{h}} g_{h}\right)\left(k_{h}\right)\right) e^{i \leq \sum_{1 \leq h \leq n-1} u_{r} k_{r} k_{h+1}},
\end{gathered}
$$

(v) $A_{s}, A_{s}^{+}$in Eq. (3) are free annihilation and creation operators on the Free Fock module $\Gamma$ characterized by the commutation relations

$$
\begin{gathered}
A\left(\alpha \otimes F_{f}\right) A^{+}\left(\beta \otimes F_{g}\right)=\langle\alpha, \beta\rangle\left(F_{f} \mid F_{g}\right), \\
\left(F_{f} \mid F_{g}\right)=\int_{\mathbb{R}^{n}} d u \int_{\mathbb{R}^{d}} d k e^{i u k p} \bar{f}(k)\left(S_{u} g\right)(k),
\end{gathered}
$$

and, if $\mathbf{p}_{1}$ denotes the momentum operator on the oneparticle space of the QEM field,

$$
\mathbf{p} A^{+}\left(\alpha \otimes F_{f}\right)=A^{+}\left(\alpha \otimes F_{\mathbf{p}_{1} f}\right) \mathbf{p} .
$$

The two relations (7) and (8) characterize the Fock module as a right Fock module over $L^{\infty}(p)$ in the sense that the scalar product

$$
\begin{gathered}
\left\langle A^{+}\left(\alpha_{1} \otimes f_{1}\right) \ldots A^{+}\left(\alpha_{n} \otimes f_{n}\right) \Phi,\right. \\
\left.A^{+}\left(\beta_{1} \otimes g_{1}\right) \ldots A^{+}\left(\beta_{n} \otimes g_{n}\right) \Phi\right\rangle
\end{gathered}
$$

computed using (8) and (7) gives exactly the expression (6).
The meaning of the limit (2) is the following. One introduces the so-called collective creation-annihilation operators:

$$
\begin{gathered}
A_{\lambda}(S, T, f) \\
:=\lambda \int_{S / \lambda^{2}} d t \int_{\mathbf{R}^{d}}\left(e^{-i t k \mathbf{p}} e^{i k \mathbf{q}} \otimes a_{k}\right) \overline{S_{t} f}(k) d k, \\
A_{\lambda}^{+}(S, T, f) \\
:=\lambda \int_{S / \lambda^{2}}^{T / \lambda^{2}} d t \int_{\mathbf{R}^{d}}\left(e^{i t k \mathbf{p}} e^{i k \mathbf{q}} \otimes a_{k}^{+}\right) S_{t} f(k) d k,
\end{gathered}
$$

where $S, T \in \mathbf{R}$ with $S \leq T$ and $f$ is a Schwartz function.

With these notations, the main result of $[3,4]$ can be stated as follows: for any $n, m \in \mathbf{N}, S_{1}, T_{1}, \ldots, S_{n}, T_{n}, S_{1}^{\prime}$, $T_{1}^{\prime}, \ldots, S_{m}^{\prime}, T_{m}^{\prime} \in \mathbf{R}, f_{1}, \ldots, f_{n}$, and Schwartz functions $f_{1}^{\prime}, \ldots, f_{m}^{\prime}$, the matrix element of the evolution operator $U_{t / \lambda^{2}}^{(\lambda)}$

$$
\begin{gathered}
\left\langle A_{\lambda}^{+}\left(S_{1}, T_{1}, f_{1}\right) \ldots A_{\lambda}^{+}\left(S_{n}, T_{n}, f_{n}\right) \Phi,\right. \\
\left.U_{t / \lambda^{2}}^{(\lambda) \dagger} A_{\lambda}^{+}\left(S_{1}^{\prime}, T_{1}^{\prime}, f_{1}^{\prime}\right) \ldots A_{\lambda}^{+}\left(S_{m}^{\prime}, T_{m}^{\prime}, f_{m}^{\prime}\right) \Phi\right\rangle
\end{gathered}
$$

converges, as $\lambda \longrightarrow 0$, to the corresponding matrix element of the evolution operator $U_{t}$ which solves the limit equation (3), i.e.,

$$
\begin{gathered}
\left\langle A^{+}\left(\chi_{\left[S_{1}, T_{1}\right]} \otimes f_{1}\right) \ldots A^{+}\left(\chi_{\left[S_{n}, T_{n}\right]} \otimes f_{n}\right) \Psi,\right. \\
\left.U^{\dagger}(t) A^{+}\left(\chi_{\left[S_{1}^{\prime}, T_{1}^{\prime}\right]} \otimes f_{1}^{\prime}\right) \ldots A^{+}\left(\chi_{\left[S_{m}^{\prime}, T_{m}^{\prime}\right]} \otimes f_{m}^{\prime}\right) \Psi\right\rangle,
\end{gathered}
$$

where $U^{\dagger}$ means $U$ or $U^{*}$ and $\Psi$ (resp. $\Phi$ ) is the vacuum vector of the QED Hilbert module $\Gamma$ ((resp. the original Fock space).

A simpler proof of this result was given in [5] and a diagrammatic approach to the result was developed in $[6,7]$.

In the following we will study the same type of convergence for the expectation values of the evolved number operator.

\section{PHOTON STATISTICS: STATEMENT OF THE PROBLEM}

In this paper, we consider the following problem: given the dynamics defined by the evolution equation (3) of Section (1.2), determine the photon statistics at time $t$ under the condition that the field was in the vacuum state at time 0 . 
Since the photon statistics is determined by the number operator, we introduce a generalized number operator of the form

$$
\begin{gathered}
N(G):=\sum_{k} G(k) a_{k}^{+} a_{k} \cong 1 \otimes \sum_{k} G(k) a_{k}^{+} a_{k} \\
\approx 1 \otimes \int_{\mathbf{R}^{d}} G(k) a_{k}^{+} a_{k}
\end{gathered}
$$

for a sufficiently regular measurable function $G$ : $\mathbb{R}^{d} \longrightarrow \mathbb{R}$ (what we need is that, for any pair of Schwartz functions $f, f^{\prime}$, the integral

$$
\left\langle F_{f^{\prime}}, T_{G} F_{f}\right\rangle:=\int_{\mathbf{R}} d \tau \int_{\mathbf{R}^{d}} d k\left(\overline{f^{\prime}} G S_{\tau} f\right)(k) e^{2 i \tau k \mathbf{p}}
$$

is a bounded operator on $\mathscr{H}_{S}$ ). Given the operator (23) as input, we define, for any time $t>0$, the forward output process as

$$
\Lambda_{+}^{(\lambda)}(t):=U_{t / \lambda^{2}}^{(\lambda)}\left(1 \otimes \sum_{k} G(k) a_{k}^{+} a_{k}\right) U_{t / \lambda^{2}}^{(\lambda) *}
$$

and the backward number process as

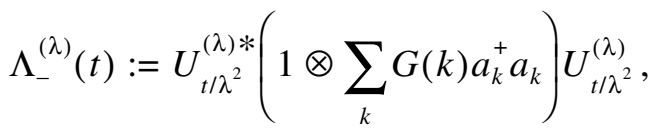

which describe the interacting radiation statistics.

\section{THE NUMBER DISTRIBUTION IN THE WCL}

The commutation rule between $A_{\lambda}^{+}(S, T, f)$ and the number operator $N(G)$ defined by (23) of Section 2 is

$$
\begin{gathered}
\left.\left[\int_{\mathbf{R}^{d}} G(k) a_{k}^{+} a_{k}\right), A_{\lambda}^{+}(S, T, f)\right] \\
=\lambda \int_{S / \lambda^{2}} d t \int_{\mathbf{R}^{d}} G(k) d k \\
\times \int_{\mathbf{R}^{d}}\left(e^{i t k^{2} \mathbf{p}} e^{i k^{\prime} \mathbf{q}} \otimes\left[a_{k}^{+} a_{k}, a_{k^{\prime}}^{+}\right]\right) S_{t} f\left(k^{\prime}\right) d k^{\prime} \\
=\lambda \int_{S / \lambda^{2}}^{T} d t \int_{\mathbf{R}^{d}}\left(e^{i t k \mathbf{p}} e^{i k \mathbf{q}} \otimes a_{k}^{+}\right) G(k) S_{t} f(k) d k .
\end{gathered}
$$

Denote by $M_{G}$ the multiplication operator, in momentum representation, by the function $G$. By the definition of the unitary group $\left\{S_{t}\right\}_{t}$ (see (5) of Section 1.2) the operator $M_{G}$ commutes with any $S_{t}$.

We will investigate the matrix elements of the number operator $N(G)$ in the collective number vectors, i.e., expressions of the form

$$
\begin{aligned}
& \left\langle\Phi, A^{\varepsilon(1)}\left(S_{1}, T_{1}, f_{1}\right) \ldots A^{\varepsilon(n)}\left(S_{n}, T_{n}, f_{n}\right)\left(\int_{\mathbf{R}^{d}} G(k) a_{k}^{+} a_{k}\right)\right. \\
& \quad \times A^{\varepsilon(n+1)}\left(S_{n+1}, T_{n+1}, f_{n+1}\right) \\
& \left.\quad \times \ldots A^{\varepsilon(n+m)}\left(S_{n+m}, T_{n+m}, f_{n+m}\right) \Phi\right\rangle,
\end{aligned}
$$

where in order to have nontrivial result, we consider $n$, $m$, and $\varepsilon$ such that

(i) $n+m=2 N$ is even;

(ii) $\varepsilon \in\{0,1\}_{+}^{2 N}$ and $|\{j \in\{1, \ldots, n\}: \varepsilon(j)=0\}|>$ $|\{j \in\{1, \ldots, n\}: \varepsilon(j)=1\}| ; \mid\{j \in\{n+1, \ldots, n+m\}:$ $\varepsilon(j)=1\}|>|\{j \in\{n+1, \ldots, n+m\}: \varepsilon(j)=0\} \mid$.

We fix two atomic vectors $\xi, \eta \in \mathscr{H}_{S}$ and we study matrix elements of the form

$$
\begin{aligned}
& \left\langle\xi,\left\langle\Phi, A_{\lambda}^{\varepsilon(1)}\left(S_{1}, T_{1}, f_{1}\right) \ldots A_{\lambda}^{\varepsilon(n)}\left(S_{n}, T_{n}, f_{n}\right)\right.\right. \\
\times & \int_{\mathbf{R}^{d}} G(k) a_{k}^{+} a_{k} d k A_{\lambda}^{\varepsilon(n+1)}\left(S_{n+1}, T_{n+1}, f_{n+1}\right)
\end{aligned}
$$

$$
\times \ldots A_{\lambda}^{\varepsilon(n+m)}\left(S_{n+m}, T_{n+m}, f_{n+m}\right) \Phi|\eta\rangle,
$$

where

(i) $\sum_{h=1}^{n} \varepsilon(h)<n / 2$;

(ii) $\sum_{h=1}^{m} \varepsilon(n+h)>m / 2$;

(iii) $n+m=2 N, \sum_{h=1}^{n+m} \varepsilon(h)=N$ for some $N$.

By the definition (9), (10) (see Section 1.2) of $A_{\lambda}^{ \pm}$, (29) is equal to 


$$
\begin{gathered}
\lambda^{n+m} \int_{S_{1} / \lambda^{2}}^{T_{1} / \lambda^{2}} d t_{1} \ldots \int_{S_{n+m} / \lambda^{2}}^{T_{n+m} / \lambda^{2}} d t_{n+m} \int_{\mathbf{R}^{(n+m) d}} d k_{1} \ldots d k_{n+m} \\
\times \prod_{h=1}^{n+m}\left(S_{t_{h}} f_{h}\right)^{\varepsilon(h)}\left(k_{h}\right) \\
\times \int_{\mathbf{R}^{d}} d k G(k)\left\langle 0\left|a_{k_{1}}^{\varepsilon(1)} \ldots a_{k_{n}}^{\varepsilon(n)} a_{k}^{+} a_{k} a_{k_{n+1}(n+1)}^{\varepsilon\left(a_{k_{n+m}}^{\varepsilon(n+m)}\right.}\right| 0\right\rangle \\
\times\left\langle\xi,\left(e^{-i t_{1} k_{1} \mathbf{p}} e^{i k_{1} \mathbf{q}}\right)^{\varepsilon(1)} \ldots\left(e^{-i t_{n+m} k_{n+m} \mathbf{p}} e^{i k_{n+m} \mathbf{q}}\right)^{\varepsilon(n+m)} \eta\right\rangle .
\end{gathered}
$$

An important step in our investigation is to understand the second scalar product of (30), i.e., denoting $2 N=$ $n+m$,

$$
\begin{gathered}
\left\langle\xi,\left(e^{-i t_{1} k_{1} \mathbf{p}} e^{i k_{1} \mathbf{q}}\right)^{\varepsilon(1)}\left(e^{-i t_{2} k_{2} \mathbf{p}} e^{i k_{2} \mathbf{q}}\right)^{\varepsilon(2)}\right. \\
\left.\times \ldots\left(e^{-i t_{2 N} k_{2 N} \mathbf{p}} e^{i k_{2 N} \mathbf{q}}\right)^{\varepsilon(2 N)} \eta\right\rangle .
\end{gathered}
$$

The CCR imply that for any $x, y$,

$$
e^{i x \mathbf{q}} e^{i y \mathbf{p}}=e^{-i x y} e^{i y \mathbf{p}} e^{i x \mathbf{q}}
$$

and this formula allows one to bring all the $e^{ \pm i k_{j} \mathbf{q}}$ in (31) near to $\eta$. Repeatedly using (32), the scalar product (31) becomes the product of

$$
\left\langle\xi, \exp \left(-i \sum_{h=1}^{2 N}(-1)^{\varepsilon(h)} t_{h} k_{h} \mathbf{p}\right) \eta\right\rangle
$$

and

$$
\begin{gathered}
\exp \left(-i \sum_{1 \leq h \leq r \leq 2 N} t_{r} k_{h} k_{r}(-1)^{1-\varepsilon(r)+\varepsilon(h)}\right. \\
\left.+i \sum_{h=1}^{2 N} t_{h} k_{h} k_{h} \delta_{1, \varepsilon(h)}\right)
\end{gathered}
$$

By the CCR, the first scalar product in (30) can also be calculated, and is equal to

$$
\begin{gathered}
\sum_{\substack{\alpha \in\{r \leq n: \varepsilon(r)=0\} \\
\beta \in\{r \leq m: \varepsilon(n+r)=1\}}} \delta_{\left(k-k_{\alpha}\right)} \delta_{\left(k-k_{\beta}\right)} \\
\times\langle 0| a_{k_{1}}^{\varepsilon(1)} \ldots \widehat{a_{k_{\alpha}}} \ldots a_{k_{n}}^{\varepsilon(n)} a_{k_{n+1}(n+1)}^{\varepsilon\left(. a_{k_{\beta}}^{+}\right.} \ldots a_{k_{n+m}(n+m)}^{\varepsilon(0\rangle,},
\end{gathered}
$$

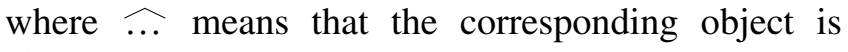
absent.
Now we introduce a shift to rewrite the $t_{j}^{\prime} s, k_{j}^{\prime} s$. Let us denote

$$
\begin{gathered}
u:=t_{\alpha}, \quad v:=t_{\beta}, \\
s_{j}:= \begin{cases}t_{j}, & \text { if } j=1,2, \ldots, \alpha-1 \\
t_{j-1}, & \text { if } j=\alpha+1, \ldots, \beta-1 \\
t_{j-2}, & \text { if } j=\beta+1, \ldots, 2 N,\end{cases} \\
S_{j}^{\prime}:= \begin{cases}S_{j}, & \text { if } j=1,2, \ldots, \alpha-1 \\
S_{j-1}, & \text { if } j=\alpha+1, \ldots, \beta-1 \\
S_{j-2}, & \text { if } j=\beta+1, \ldots, 2 N,\end{cases} \\
T_{j}^{\prime}:= \begin{cases}T_{j}, & \text { if } j=1,2, \ldots, \alpha-1 \\
T_{j-1}, & \text { if } \quad j=\alpha+1, \ldots, \beta-1 \\
T_{j-2}, & \text { if } \quad j=\beta+1, \ldots, 2 N,\end{cases} \\
f:=f_{\alpha}, \quad g:=f_{\beta}
\end{gathered}
$$$$
g_{j}:=\left\{\begin{array}{l}
f_{j}, \quad \text { if } \quad j=1,2, \ldots, \alpha-1 \\
f_{j-1}, \quad \text { if } \quad j=\alpha+1, \ldots, \beta-1 \\
f_{j-2}, \quad \text { if } \quad j=\beta+1, \ldots, 2 N,
\end{array}\right.
$$$$
z_{j}:=\left\{\begin{array}{l}
k_{j}, \quad \text { if } j=1,2, \ldots, \alpha-1 \\
k_{j-1}, \quad \text { if } j=\alpha+1, \ldots, \beta-1 \\
k_{j-2}, \quad \text { if } \quad j=\beta+1, \ldots, 2 N
\end{array}\right.
$$

and

$$
\varepsilon_{\alpha, \beta}(j):= \begin{cases}\varepsilon(j)_{j}, \quad \text { if } \quad j=1,2, \ldots, \alpha-1 \\ \varepsilon(j-1), \quad \text { if } j=\alpha+1, \ldots, \beta-1 \\ \varepsilon(j-2), \quad \text { if } j=\beta+1, \ldots, 2 N .\end{cases}
$$

With these notations, we know that

$$
\begin{gathered}
\left\langle 0\left|a_{k_{1}}^{\varepsilon(1)} \ldots \widehat{a}_{k_{\alpha}} \ldots a_{k_{n}}^{\varepsilon(n)} a_{k_{n+1}}^{\varepsilon(n+1)} \ldots \widehat{a}_{k_{\beta}}^{+} \ldots a_{k_{n+m}}^{\varepsilon(n+m)}\right| 0\right\rangle \\
=\left\langle 0\left|a_{z_{1}, \beta}^{\varepsilon_{\alpha \beta}(1)} \ldots a_{z_{2 N}}^{\varepsilon_{\alpha \beta}(2 N-2)}\right| 0\right\rangle, \\
\quad-i \sum_{h=1}^{2 N}(-1)^{\varepsilon(h)} t_{h} k_{h} \mathbf{p} \\
=-i \sum_{h=1}^{2 N-2}(-1)^{\varepsilon_{\alpha, \beta}(h)} z_{h} \mathbf{p}^{-i u k} \mathbf{p}^{+i v k} \mathbf{p},
\end{gathered}
$$




$$
\begin{gathered}
=\sum_{1 \leq h<r \leq 2 N-2} s_{r} z_{h} z_{r}(-1)^{1-\varepsilon_{\alpha, \beta}(r)+\varepsilon_{\alpha, \beta}(h)} \\
+V \sum_{h=\alpha}^{\beta-2} z_{h} k(-1)^{\varepsilon_{\alpha, \beta}(h)}+\sum_{h=\beta-1}^{2 N-2} s_{h} z_{h} k(-1)^{\varepsilon_{\alpha, \beta}(h)} \\
-\sum_{h=\alpha}^{2 N-2} s_{h} z_{h} k(-1)^{\varepsilon_{\alpha, \beta}(h)}+V \sum_{h=1}^{\alpha-1} z_{h} k(-1)^{\varepsilon_{\alpha, \beta}(h)} \\
\quad-u \sum_{h=1}^{\alpha-1} z_{h} k(-1)^{\varepsilon_{\alpha, \beta}(h)}-v k k \\
:=\sum_{1 \leq h<r \leq 2 N-2} s_{r} z_{h} z_{r}(-1)^{1-\varepsilon_{\alpha, \beta}(r)+\varepsilon_{\alpha, \beta}(h)} \\
+\mathscr{T}\left(\left\{s_{h^{\prime}} z_{h}\right\}_{h=1}^{2 N-2}, \varepsilon, \alpha, \beta, u, v, k\right)-v k k
\end{gathered}
$$

and

$$
\sum_{h=1}^{2 N} t_{h} k_{h} k_{h} \delta_{1, \varepsilon(h)}=v k k+\sum_{h=1}^{2 N-2} s_{h} z_{h} z_{h} \delta_{1, \varepsilon_{\alpha, \beta}(h)} .
$$

So, (30) is equal to

$$
\begin{aligned}
& \sum_{\substack{\alpha \in\{r \leq n: \varepsilon(r)=0\} \\
\beta \in\{r \leq m: \varepsilon(n+r)=1\}}} \lambda^{2} \int_{S_{\alpha} / \lambda^{2}}^{T_{\alpha} / \lambda^{2}} d u \int_{S_{\beta} / \lambda^{2}}^{T_{\beta} / \lambda^{2}} d v \\
& \times \int_{\mathbf{R}^{d}} d k\left(G \overline{\left(S_{u} f_{\alpha}\right)} S_{V} f_{\beta}\right)(k) \lambda^{2 N-2} \int_{S_{1}^{\prime} / \lambda^{2}}^{T_{1}^{\prime} / \lambda^{2}} d s_{1} \\
& T_{2 N-2}^{\prime} / \lambda^{2} \\
& \times \ldots \int_{S_{2 N-2}^{\prime} / \lambda^{2}} d s_{2 N-2} \int_{\mathbf{R}^{(2 N-2) d}} d z_{1} \ldots d z_{2 N-2} \\
& \times \prod_{h=1}^{2 N-2}\left(S_{s_{h}} g_{h}\right)^{\varepsilon_{\alpha, \beta}(h)}\left(z_{h}\right)\left\langle 0\left|a_{z_{1}}^{\varepsilon_{\alpha, \beta}(1)} \ldots a_{z_{2 N-2}}^{\varepsilon_{\alpha, \beta}(2 N-2)}\right| 0\right\rangle \\
& \times\left\langle\xi, \exp \left(-i \sum_{h=1}^{2 N-2}(-1)^{\varepsilon_{\alpha, \beta}(h)} z_{h} \mathbf{p}-i u k \mathbf{p}+i v k \mathbf{p}\right) \eta\right\rangle \\
& \times \exp \left(-i \sum_{1 \leq h<r \leq 2 N-2} s_{r} z_{h} z_{r}(-1)^{1-\varepsilon_{\alpha, \beta}(r)+\varepsilon_{\alpha, \beta}(h)}\right. \\
& \left.+i \sum_{h=1}^{2 N-2} s_{h} z_{h} z_{h} \delta_{1, \varepsilon_{\alpha, \beta}(h)}\right) \\
& \times \exp \left(-i \mathscr{T}\left(\left\{s_{h^{\prime}} z_{h}\right\}_{h=1}^{2 N-2}, \varepsilon, \alpha, \beta, u, v, k\right)\right) \text {. }
\end{aligned}
$$

Comparing with [4], what is really new is the factor $\mathscr{T}\left(\left\{s_{h^{\prime}} z_{h}\right\}_{h=1}^{2 N-2}, \varepsilon, \alpha, \beta, u, v, k\right)$, which according to the definition, is equal to

$$
\begin{aligned}
& V \sum_{h=\alpha}^{\beta-2} z_{h} k(-1)^{\varepsilon_{\alpha, \beta}(h)}+\sum_{h=\beta-1}^{2 N-2} s_{h} z_{h} k(-1)^{\varepsilon_{\alpha, \beta}(h)} \\
& -\sum_{h=\alpha}^{2 N-2} s_{h} z_{h} k(-1)^{\varepsilon_{\alpha, \beta}(h)}+V \sum_{h=1}^{\alpha-1} z_{h} k(-1)^{\varepsilon_{\alpha, \beta}(h)} \\
& -u \sum_{h=1}^{\alpha-1} z_{h} k(-1)^{\varepsilon_{\alpha, \beta}(h)}=V \sum_{h=\alpha}^{\beta-2} z_{h} k(-1)^{\varepsilon_{\alpha, \beta}(h)} \\
& -\sum_{h-2} s_{h} z_{h} k(-1)^{\varepsilon_{\alpha, \beta}(h)}+(V-u) \sum_{h=1}^{\alpha-1} z_{h} k(-1)^{\varepsilon_{\alpha, \beta}(h)} .
\end{aligned}
$$

Since there is no $z_{h} z_{r}$-factor in the term $\mathscr{T}\left(\left\{s_{h^{\prime}} z_{h}\right\}_{h=1}^{2 N-2}\right.$, $\varepsilon, \alpha, \beta, u, v, k)$, we can repeat the discussion of Sections 4 and 6 of [4] to find the limit of (45). This gives the following result:

Lemma 1. If $\varepsilon \in\{0,1\}_{-}^{2 N}$ (this implies that for any $\alpha \in\{r \leq n: \varepsilon(r)=0\}$ and $\beta \in\{n \leq r \leq n+m: \varepsilon(r)=1\}$, $\varepsilon_{\alpha, \beta}$ defined as before is in $\left.\{0,1\}_{-}^{2 N-2}\right)$, the limit of (45), as $\lambda \longrightarrow 0$, exists and is equal to zero

Now we consider an $\varepsilon \in\{0,1\}_{+}^{2 N}$ (with $2 N=n+m$ ). It is known (see [4] for the proof) that there is exactly one noncrossing pair partition, say $\left\{l_{h}, r_{h}\right\}_{h=1}^{N}$ of the set $\{1,2, \ldots, 2 N\}$, such that

$$
\begin{gathered}
\left\{l_{h}\right\}_{h=1}^{N}=\{j \in\{1, \ldots, 2 N\}: \varepsilon(j)=0\} ; \\
\left\{r_{h}\right\}_{h=1}^{N}=\{j \in\{1, \ldots, 2 N\}: \varepsilon(j)=1\} ; \\
l_{1}<l_{2}<\ldots<l_{N} ; \quad l_{h}<r_{h}, \quad \forall h=1, \ldots, N .
\end{gathered}
$$

Now we introduce some new notions about pair partitions.

Definition 1. A pair $(\alpha, \beta)$ is said to be admissible for $\left\{l_{h}, r_{h}\right\}_{h=1}^{N}$ if there is an $h=1,2, \ldots, N$, such that $\alpha=l_{h}$ and $\beta=r_{h}$. For a given $n \leq 2 N-1,(\alpha, \beta)$ is said to be the minimum admissible pair of $\left\{l_{h}, r_{h}\right\}_{h=1}^{N}$ around $n$, if

(i) $(\alpha, \beta)$ is admissible to $\left\{l_{h}, r_{h}\right\}_{h=1}^{N}$;

(ii) $\alpha \leq n, \beta \geq n+1$;

(iii) for any for any $j=1, \ldots, N$, if $l_{j}>\alpha$ (it follows form the non-crossing principle that $r_{j}<\beta$ ), then either $r_{j} \leq n$ or $l_{j} \geq n+1$.

Remark 1. Clearly, for each $N \in \mathbf{N}, n=1,2, \ldots 2 N-1$ and a noncrossing pair partition $\left\{l_{h}, r_{h}\right\}_{h=1}^{N}$, there

OPTICS AND SPECTROSCOPY Vol. $103 \quad$ No. 22007 
exists a unique minimum admissible pair of $\left\{l_{h}, r_{h}\right\}_{h=1}^{N}$ around $n$.

Lemma 2. For any $N \in \mathbf{N}, \varepsilon \in\{0,1\}_{+}^{2 N}$ which determines the noncrossing pair partition $\left\{l_{h}, r_{h}\right\}_{h=1}^{N}$ and $\alpha, \beta \in\{1,2, \ldots, 2 N\}$ (with $\alpha<\beta$ ). Define

$$
\begin{gathered}
l_{j}^{(\alpha, \beta)}:= \begin{cases}l_{j}, \quad \text { if } j=1,2, \ldots, \alpha-1 \\
l_{j-1}, \quad \text { if } j=\alpha+1, \ldots, \beta-1 \\
l_{j-2}, \quad \text { if } j=\beta+1, \ldots, 2 N,\end{cases} \\
r_{j}^{(\alpha, \beta)}:= \begin{cases}r_{j}, \quad \text { if } j=1,2, \ldots, \alpha-1 \\
r_{j-1}, \text { if } j=\alpha+1, \ldots, \beta-1 \\
r_{j-2}, \text { if } j=\beta+1, \ldots, 2 N .\end{cases}
\end{gathered}
$$

Then, $\left\{l_{h}^{(\alpha, \beta)}, r_{h}^{(\alpha, \beta)}\right\}_{h=1}^{N}$ is the noncrossing pair partition of the set $\{1,2, \ldots, 2 N-2\}$ determined by $\varepsilon_{\alpha, \beta} \in\{0,1\}_{+}^{2 N-2}$ if $(\alpha, \beta)$ is admissible for $\left\{l_{h}, r_{h}\right\}_{h=1}^{N}$.

Proof. A trivial calculation.

Again, thanks to the fact that there is no $z_{h} z_{r}$-factor in

$$
\mathscr{T}\left(\left\{s_{h^{\prime}} z_{h}\right\}_{h=1}^{2 N-2}, \varepsilon, \alpha, \beta, u, v, k\right)
$$

the contributions to the limit (45) of the factor

$$
\left\langle 0\left|a_{z_{1}, \beta}^{\varepsilon_{\alpha, \beta}(1)} \ldots a_{z_{2 N-2}}^{\varepsilon_{\alpha, \beta}(2 N-2)}\right| 0\right\rangle
$$

are (as argued in [4]) zero except for the term determined by the noncrossing pair partition

$$
\left\{l_{h}^{(\alpha, \beta)}, r_{h}^{(\alpha, \beta)}\right\}_{h=1}^{N} .
$$

That is, the only possibly nonzero contribution if the factor

$$
\mathscr{T}\left(\left\{s_{h^{\prime}} z_{h}\right\}_{h=1}^{2 N-2}, \varepsilon, \alpha, \beta, u, v, k\right)
$$

could be forgotten and has the form

$$
\begin{aligned}
& \sum_{\substack{\alpha \in\{r \leq n: \varepsilon(r)=0\} \\
\beta \in\{r \leq m: \varepsilon(n+r)=1\}}}\left\langle\chi_{\left[S_{\alpha}, T_{\alpha}\right]}, \chi_{\left[S_{\beta}, T_{\beta}\right]}\right\rangle
\end{aligned}
$$

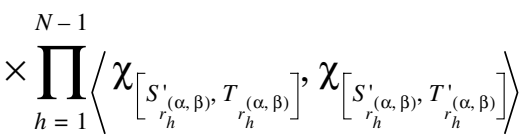

$$
\begin{aligned}
& \times \int_{\mathbf{R}^{N}} d \tau d \tau_{1} \ldots d \tau_{N-1} \int_{\mathbf{R}^{N d}} d k d k_{1} \ldots d k_{N-1}\left(\bar{f}_{\alpha} G S_{\tau} f_{\beta}\right)(k) \\
& \times \prod_{h=1}^{N-1}\left(\overline{g_{l_{h}^{(\alpha, \beta}}} S_{\tau_{h}} g_{r_{h}^{(\alpha, \beta)}}\right)\left(k_{h}\right)
\end{aligned}
$$

$$
\begin{aligned}
& \times\left\langle\xi, \exp \left(i \sum_{h=1}^{N-1} \tau_{h} k_{h} \mathbf{p}+i \tau k \mathbf{p}\right) \eta\right\rangle \\
\times & \exp \left(i \sum_{1 \leq x<y \leq N-1} \tau_{x} k_{x} k_{y} \chi_{\left(l_{y}, r_{y}\right)}\left(l_{x}\right)\right),
\end{aligned}
$$

where the following changes of variables have been performed:

$$
\begin{gathered}
k_{x}:=z_{r_{x}^{(\alpha, \beta)}}, \quad \forall x=1,2, \ldots, N-1, \\
u^{\prime}:=\lambda^{2} u ; \quad \tau:=v-u^{\prime} / \lambda^{2} ; \quad s_{x}^{\prime}:=\lambda^{2} s_{l_{x}^{(\alpha, \beta)}} ; \\
\tau_{x}:=s_{r_{x}^{(\alpha, \beta)}}-s_{x}^{\prime} / \lambda^{2}, \quad \forall x=1,2, \ldots, N-1 .
\end{gathered}
$$

For this, possibly nonzero, contribution, we have also to consider the role of the factor

$$
\mathscr{T}\left(\left\{s_{h^{\prime}} z_{h}\right\}_{h=1}^{2 N-2}, \varepsilon, \alpha, \beta, u, v, k\right) .
$$

This consideration does not eliminate our unique possibly nonzero, term only if under the changes of variables (52), the factor $\mathscr{T}\left(\left\{s_{h} z_{h}\right\}_{h=1}^{2 N-2}, \varepsilon, \alpha, \beta, u, v, k\right)$ could be represented as a function of $\tau, k,\left\{\tau_{x}, k_{x}\right\}_{x=1}^{N}$.

Lemma 3. The quantity $\mathscr{T}\left(\left\{s_{h^{\prime}} z_{h}\right\}_{h=1}^{2 N-2}, \varepsilon, \alpha, \beta, u\right.$, $\checkmark, k)$ can be represented as a function of $\tau, k$, $\left\{\tau_{x}, k_{x}\right\}_{x=1}^{N}$ if $(\alpha, \beta)$ is the minimum admissible pair of $\left\{l_{h}, r_{h}\right\}_{h=1}^{N}$ around $n$.

Theorem 1. The limit, as $\lambda \longrightarrow 0$, of (30) exists and is equal to

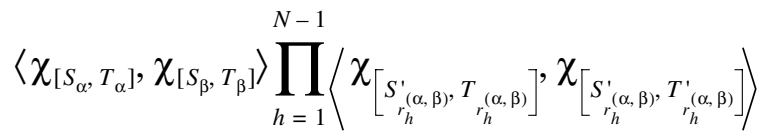

$$
\begin{aligned}
& \times \int_{\mathbf{R}^{N}} d \tau d \tau_{1} \ldots d \tau_{N-1} \int_{\mathbf{R}^{N_{d}}} d k d k_{1} \ldots d k_{N-1}\left(\overline{f_{\alpha}} G S_{\tau} f_{\beta}\right)(k) \\
& \times \prod_{h=1}^{N-1}\left(\overline{g_{l_{h}^{(\alpha, \beta)}}} S_{\tau_{h}} g_{r_{h}^{(\alpha, \beta)}}\right)\left(k_{h}\right) \\
& \times\left\langle\xi, \exp \left(i \sum_{h=1}^{N-1} \tau_{h} k_{h} \mathbf{p}+i \tau k \mathbf{p}\right) \eta\right\rangle \\
& \times \exp \left(i \sum_{1 \leq x<y \leq N-1} \tau_{x} k_{x} k_{y} \chi_{\left(l_{y}, r_{y}\right)}\left(l_{x}\right)\right) \exp \left(i \sum_{h=h_{0}}^{j_{0}-1} \tau_{h} k_{h} k\right),
\end{aligned}
$$

where $\{\alpha, \beta\}=\left\{l_{h_{0}}, r_{h_{0}}\right\}$ is the minimum admissible pair of $\left\{l_{h}, r_{h}\right\}_{h=1}^{N}$ around $n ;\left\{l_{h}, r_{h}\right\}_{h=h_{0}}^{j_{0}}$ is the restric- 
tion of the noncrossing pair partition $\left\{l_{h}, r_{h}\right\}_{h=1}^{N}$ on the set $\{\alpha, \alpha+1, \ldots, \beta\}$.

Theorem 2. As $\lambda \longrightarrow 0$, the limits of the forward (36) and the backward (II) output number operators exist. Moreover, the limit of the forward output number operator (36) is equal to

$$
\Lambda_{+}(t):=U^{*}(t)\left(1 \otimes N\left(T_{G}\right)\right) U(t)
$$

and the limit of the backward output number operator (37) is

$$
\Lambda_{-}(t):=U(t)\left(1 \otimes N\left(T_{G}\right)\right) U^{*}(t),
$$

where $N\left(T_{G}\right)$ is the usual number operator on the Free Fock module $\Gamma$, which is defined

for any $n \in \mathbf{N}, \varepsilon \in\{0,1\}^{n}$, such that $\forall k=1,2, \ldots, n$,

$$
\begin{gathered}
|\{j=k, k+1, \ldots, n: \varepsilon(j)=1\}| \\
\geq|\{j=k, k+1, \ldots, n: \varepsilon(j)=0\}|, \\
N\left(T_{G}\right) A^{+}\left(\chi_{1} \otimes F_{f_{1}}\right) \ldots A^{+}\left(\chi_{n} \otimes F_{f_{n}}\right) \\
:=A^{+}\left(\chi_{1} \otimes T_{G} F_{f_{1}}\right) \ldots A^{+}\left(\chi_{n} \otimes F_{f_{n}}\right),
\end{gathered}
$$

and

$$
N\left(T_{G}\right) B=B N\left(T_{G}\right):=N\left(B T_{G}\right), \quad \forall B \in \mathscr{P} .
$$

Remark 2. Notice that in (40), we have e $e^{2 i \tau k p}$ but not $e^{i \tau k \mathbf{p}}$, since the number operator also contributes a factor $e^{i \tau k \mathbf{p}}$.

\section{MEAN VALUES}

\section{OF OUTPUT NUMBER OPERATORS}

Denote, for any atomic state $\xi$ ( $\Psi$ being the vacuum state after the stochastic limit),

$V\left(t, T_{G}\right):=\left\langle\xi,\left\langle\Psi,\left(U^{*}(t)\left(1 \otimes N\left(T_{G}\right)\right) U(t)\right) \Psi\right\rangle \xi\right\rangle$,

using the techniques of quantum stochastic calculus on the QED module [8], one has

$$
\begin{aligned}
& V\left(t, T_{G}\right)=\left\langle\xi,\left\langle\Psi, \sum_{n=0}^{\infty} \sum_{\epsilon \in\{0, \pm 1\}^{n} 0} \int_{t_{1}}^{t} d a_{0}^{\epsilon(1)} d a_{t_{2}}^{\epsilon(2)}\right.\right. \\
& \times \ldots \int_{0}^{t_{1}} d a_{t_{2}}^{\epsilon(n)}\left(1 \otimes \mathcal{N}\left(T_{G}\right)\right) \\
& \times \sum_{m=0}^{\infty} \sum_{\sigma \in\{0, \pm 1\}^{m}} \int_{0}^{t} d b_{s_{1}}^{\sigma(1)} \int_{0}^{s_{1}} d b_{s_{2}}^{\sigma(2)} \ldots \int_{0}^{s_{m-1}} d b_{s_{m}}^{\sigma(m)} \Psi|\xi\rangle,
\end{aligned}
$$

where

$$
\begin{aligned}
& d a_{u}^{\epsilon}:=\left\{\begin{array}{l}
-d A_{u}^{+}\left(F_{g}\right)(-i \mathbf{p}), \text { if } \epsilon=1 \\
(-i \mathbf{p})^{+} d A_{u}\left(F_{g}\right), \text { if } \epsilon=-1 \\
X_{+} d u, \text { if } \epsilon=0,
\end{array}\right. \\
& d b_{V}^{\sigma}:=\left\{\begin{array}{l}
d A_{V}^{+}\left(F_{g}\right)(-i \mathbf{p}), \text { if } \sigma=1 \\
-(-i \mathbf{p})^{+} d A_{V}\left(F_{g}\right), \text { if } \sigma=-1 \\
X_{-} d u, \text { if } \sigma=0,
\end{array}\right.
\end{aligned}
$$

and

$$
\begin{gathered}
X_{+}:=-(-i \mathbf{p})^{+}\left(F_{g} \mid F_{g}\right)_{-+}(-i \mathbf{p})=-p^{2}\left(F_{g} \mid F_{g}\right)_{-}, \\
X_{-}:=-(-i \mathbf{p})\left(F_{g} \mid F_{g}\right)_{--}(-i \mathbf{p})=p^{2}\left(F_{g} \mid F_{g}\right)_{-} .
\end{gathered}
$$

Lemma 4. In (59) the sum $\sum_{\epsilon \in\{0, \pm 1\}^{n}}$ (resp. $\sum_{\sigma \in\{0, \pm 1\}^{m}}$ can be replaced by the sum $\sum_{\epsilon \in\{0,-1\}^{n}}$ (resp. $\sum_{\sigma \in\{0,+1\}^{m}}$ ).

Proof. If $\epsilon(k)=+1$, then the operator $d a_{t_{k}}^{\epsilon(k)}=$ $-d A_{t_{k}}^{+}\left(F_{g}\right)(-i \mathbf{p})$ must act on the vacuum state which gives zero. So we can replace the sum $\sum_{\epsilon \in\{0, \pm 1\}^{n}}$ by the sum $\sum_{\epsilon \in\{0,-1\}^{n}}$. Similarly, the sum $\sum_{\sigma \in\{0, \pm 1\}^{m}}$ can be replaced by the sum $\sum_{\sigma \in\{0,+1\}^{m}}$.

Lemma 5. Denote

$$
\begin{gathered}
:=\left\langle\xi,\left\langle\Psi, \int_{0}^{t} d a_{t_{1}}^{\epsilon(1)} \int_{0}^{t_{1}} d a_{t_{2}}^{\epsilon(2)} \ldots \int_{0}^{t_{n-1}} d t a_{t_{2}}^{\epsilon(n)}\left(1 \otimes \mathcal{N}\left(T_{G}\right)\right)\right.\right. \\
\left.\left.\quad \times \int_{0}^{t} d b_{s_{1}}^{\sigma(1)} \int_{0}^{s_{1}} d b_{s_{2}}^{\sigma(2)} \ldots \int_{0}^{s_{m-1}} d b_{s_{m}}^{\sigma(m)} \Psi\right\rangle\right)
\end{gathered}
$$

Then

(i) $V_{0, n}\left(t, T_{G} ; \epsilon, \sigma\right)$ and $V_{n, 0}\left(t, T_{G} ; \epsilon, \sigma\right)$ are zero;

(ii) for $n, m \geq 1, V_{n, m}\left(t, T_{G}\right.$; $\left.\epsilon, \sigma\right)=0$ if

$$
\left(1+\sum_{h=1}^{n} \epsilon(h)\right)^{2}+\left(1-\sum_{h=1}^{n} \sigma(h)\right)^{2}=0
$$

\section{is not verified.}

Proof.

(i) In the term $V_{0, m}\left(t, T_{G} ; \epsilon, \sigma\right)\left(\right.$ resp. $V_{n, 0}\left(t, T_{G} ; \epsilon, \sigma\right)$, we have always a quantity like $\left\langle\Psi,\left(1 \otimes \mathcal{N}\left(T_{G}\right)\right) \gamma\right\rangle$ (resp. 
$\left.\left\langle\gamma, 1 \otimes \mathcal{N}\left(T_{G}\right) \Psi\right\rangle\right)$ with $\gamma \in \Gamma$. Therefore, $V_{0, m}\left(t, T_{G}\right.$; $\epsilon, \sigma)=V_{n, 0}\left(t, T_{G} ; \epsilon, \sigma\right)=0$.

(ii) If (64) is not true, then we have, in the expression $V_{n, m}\left(t, T_{G} ; \epsilon, \sigma\right)$,

(iia) more $d A$ less $d A^{+}$, or

(iib) more $d A^{+}$less $d A$, or

(iic) $r^{\prime} s d A$ (in the left hand side of the number operator) and $r^{\prime} s d A^{+}$(in the right hand side of the number operator) with $r \geq 2$.

In the cases (iia) and (iib), we have always an annihilator acting on the vacuum vector, which gives zero. In order to treat the case (iic), denote the time variables of the $r$ 's $d A$ (resp. the $r$ 's $d A^{+}$) as $t_{h_{1}}>\ldots>t_{h_{r}}$ (resp. $s_{j_{1}}>\ldots>s_{j_{r}}$ ). Since we are considering the vacuum state, in order to be different from zero, all operators must be "used" to produce inner products among themselves (by mean of the Ito formula). By the noncrossing principle, denote $h:=h_{r}, j:=j_{1}$; the annihilator $d A_{t_{h}}(g)$ should be paired with the creator $d A_{s_{j}}^{+}\left(F_{g}\right)$. Thus, by the Ito formula, for such $n, m$ and $\epsilon, \sigma$,

$$
\begin{aligned}
& :=\left\langle\xi,\left\langle\Psi, \int_{0}^{t} d a_{t_{1}}^{\epsilon(1)} \int_{0}^{t_{1}} d a_{t_{2}}^{\epsilon(2)} \cdots \int_{0}^{t_{h-2}} d a_{t_{h-1}}^{\epsilon(h-1)} \int_{0}^{t_{h-1}} \frac{t_{h}^{n-h}}{(n-h) !}\right.\right. \\
& \quad \times \int_{0}^{t} d s_{1} \ldots \int_{0}^{s_{j-2}} d s_{j-1} \int_{0}^{s_{j-1}}(-i \mathbf{p})^{+} d A_{t_{h}}\left(F_{g}\right)
\end{aligned}
$$

$$
\begin{gathered}
\times X_{+}^{n-h}\left(1 \otimes \mathcal{N}\left(T_{G}\right)\right) X_{-}^{j-1} d A_{s_{j}}^{+}\left(F_{g}\right)(-i \mathbf{p}) \\
\times \int_{0}^{s_{j}} d b_{s_{j+1}}^{\sigma(j+1)} \ldots \int_{0}^{t_{m-1}} d b_{s_{m}}^{\sigma(m)} \Psi|\xi\rangle .
\end{gathered}
$$

By the Ito formula and the property of the number operator, we know that, for any choice of the functions $M_{1}(t), M_{2}(t)$, one has

$$
\begin{gathered}
\int_{0}^{t_{h-1} s_{j-1}} \int_{0} M_{1}\left(t_{h}\right)(-i \mathbf{p})^{+} d A_{t_{h}}\left(F_{g}\right) \\
\times X_{+}^{n-h}\left(1 \otimes \mathcal{N}\left(T_{G}\right)\right) X_{-}^{j-1} d A_{s_{j}}^{+}\left(F_{g}\right)(-i \mathbf{p}) M_{2}\left(s_{j}\right) \\
=\int_{0}^{t_{h-1} \wedge s_{j-1}} M_{1}(\tau)(-i \mathbf{p})^{+}\left(F_{g} \mid X_{+}^{n-h} T_{G} X_{-}^{j-1} F_{g}\right)(-i \mathbf{p}) \\
\times M_{2}(\tau) d \tau .
\end{gathered}
$$

So, for any $x, y \leq r, t_{h_{x}}$ should be larger than $s_{j_{y}}$ since $t_{h_{x}}>\tau>s_{j_{y}}$. The consequence of this fact is that $d A_{t_{h_{x}}}$ commutes essentially with $d A_{s_{j_{y}}}^{+}$. In the stated case, we have at least one annihilator acting on the vacuum vector giving zero.

Theorem 3. In the notations (61), (62) one has

$$
\begin{aligned}
V\left(t, T_{G}\right) & =\sum_{n, m=1}^{\infty} \sum_{1 \leq h \leq n, 1 \leq j \leq m}\left\langle\xi, X_{+}^{h-1}(-i \mathbf{p})^{+}\left(F_{g} \mid X_{+}^{n-h} T_{G} X_{-}^{j-1} F_{g}\right)(-i \mathbf{p}) X_{-}^{m-j} \xi\right\rangle \\
& \times \frac{\int_{0}^{t} d t_{1} \int_{0}^{t_{1}} d t_{2} \ldots \int_{0}^{t_{h-2}} d t_{h-1} \int_{0}^{t} d s_{1} \int_{0}^{s_{1}} d s_{2} \ldots \int_{0}^{s_{j-2}} d s_{j-1}\left(t_{h-1} \wedge s_{j-1}\right)^{n+m+1-h-j}}{(n+m+1-h-j)(n-h) !(m-j) !}
\end{aligned}
$$

Denote, for each $n, m, N \in \mathbf{N}, n, m \geq 2$,

$$
\begin{aligned}
c_{n, m}(t, N) & :=\int_{0}^{t} d t_{1} \int_{0}^{t_{1}} d t_{2} \ldots \int_{0}^{t_{n-1}} d t_{n} \int_{0}^{t} d s_{1} \int_{0}^{s_{1}} d s_{2} \\
& \times \ldots \int_{0}^{s_{m-1}} d s_{m}\left(t_{n} \wedge s_{m}\right)^{N},
\end{aligned}
$$

then

$$
V\left(t, T_{G}\right)=\sum_{n, m=1}^{\infty} \sum_{1 \leq h \leq n, 1 \leq j \leq m} X_{+}^{h-1}(-i \mathbf{p})^{+}
$$

$$
\begin{aligned}
& \left(F_{g} \mid X_{+}^{n-h} T_{G} X_{-}^{j-1} F_{g}\right)(-i \mathbf{p}) X_{-}^{m-j} \\
& \times \frac{c_{h-1, j-1}(t, n+m+1-h-j)}{(n+m+1-h-j)(n-k) !(m-j) !} .
\end{aligned}
$$

Remark 3. The forward output number operator can be investigated similarly. In fact, if in the above discussion we exchange $X_{+}$with $X_{-}$, the forward output operator is obtained. In the following section we will complete our calculation of the expectation value (58) of Section 4 by explicitly computing the coefficients $c_{m, n}(t, N)$. 


\section{THE EXPLICIT FORM \\ OF THE $c_{m, n}(t, N)$}

From the above discussion, we know that in order to determine completely the output operators, we have to determine the explicit form of the coefficients $\left\{c_{m, n}(t\right.$, $N)$ \}. This section is devoted to this purpose.

Lemma 6. The coefficients $c_{m, n}(t, N)$ (cf. (11) of Section 4) satisfy the relations

$$
\begin{gathered}
c_{n, m}(t, N)=c_{m, n}(t, N), \quad \forall n, m \in \mathbf{N}, \\
\frac{d}{d t} c_{n, m}(t, N)=c_{n-1, m}(t, N)+c_{n, m-1}(t, N), \\
c_{n, m}(0, N)=0, \quad c_{n, 0}(t, N)=\frac{N ! t^{N+n}}{(N+n) !}, \\
c_{n, 1}(t, N)=\frac{(n+1) n t^{N+n+1}}{(N+1)(N+2) \ldots(N+n+1)} . \\
n \geq 2 .
\end{gathered}
$$

Proof (70) and (71) are obtained by direct calculation. The equality (72) can be obtained as follows. First notice that

$$
\begin{gathered}
c_{n, 1}(t, N)=c_{1, n}(t, N) \\
=\int_{0}^{t} d s_{1} \int_{0}^{s_{1}} d s_{2} \ldots \int_{0}^{s_{n-1}} d s_{n} \int_{0}^{t} d t_{1}\left(t_{1} \wedge s_{n}\right)^{N} .
\end{gathered}
$$

Denoting

$$
\int_{t \geq s_{1} \geq \ldots \geq s_{m-1}} d s_{1} d s_{m}^{k} \ldots d s_{m}=: I_{m}(k),
$$

then one has

$$
I_{m}(k)=t^{k+m} \frac{k !}{(k+m) !} .
$$

Proof. In fact,

$$
\begin{gathered}
I_{m}(k)=\int_{t \geq s_{1} \geq \ldots \geq s_{m-2}} \frac{s_{m-1}^{k+1}}{k+1} d s_{1} \ldots d s_{m-1} \\
=I_{m-1}(k+1) \frac{1}{k+1}=I_{m-2}(k+2) \frac{1}{(k+2)(k+1)} \\
=\ldots=I_{m-\alpha}(k+\alpha) \frac{k !}{(k+\alpha) !} \\
=I_{1}(k+m-1) \frac{k !}{(k+m-1) !}
\end{gathered}
$$

and, thus,

$$
I_{1}(v)=\int_{0}^{t} d s_{1} s_{1}^{v}=\frac{t^{v+1}}{v+1} .
$$

Consider that the integral (73) follows

$$
\begin{gathered}
\int_{0}^{t} d t_{1}\left(t_{1} \wedge s\right)^{N}=\int_{0}^{s} d t_{1} t_{1}^{N}+s^{N} \int_{s}^{t} d t_{1} \\
=\left.\frac{t^{N+1}}{N+1}\right|_{0} ^{s}+s^{N}(t-s)=\frac{s^{N+1}}{N+1}+s^{N} t-s^{N+1} \\
=s^{N+1}\left(\frac{1}{N+1}-1\right)+s^{N} t \\
=-\frac{N}{N+1} s^{N+1}+s^{N} t=s^{N}\left(t-\frac{N}{N+1} s\right) .
\end{gathered}
$$

This implies that, form $m \geq 2$,

$$
\begin{gathered}
c_{1, m}(t, N):=\int_{0}^{t} d s_{1} \int_{0}^{s_{1}} d s_{2} \ldots \int_{0}^{s_{m-1}} d s_{m}\left(s_{1} \wedge s_{m}\right)^{N} \\
=\int_{t \geq s_{1} \geq \ldots \geq s_{m-1}} d s_{1} \ldots d s_{m}\left(s_{m}^{N} t-\frac{N}{N+1} s_{m}^{N+1}\right) \\
=t \frac{t^{N+m} N !}{(N+m) !}-\frac{N}{N+1} t^{N+1+m} \frac{(N+1) !}{(N+1+m) !} \\
=t^{N+m+1} \frac{N !}{(N+m) !}\left[1-\frac{N(N+1)}{(N+1+m)(N+1)}\right] \\
=t^{N+m+1} \frac{N !}{(N+m) !}
\end{gathered}
$$

$$
\begin{gathered}
\times\left[\frac{N^{2}+N+N+1+m N+m-N^{2}-N}{(N+1+m)(N+1)}\right] \\
=(\ldots)\left[\frac{N+1+m N+m}{(N+1+m)(N+1)}\right]
\end{gathered}
$$

$$
\begin{gathered}
=t^{N+m+1} \frac{N !}{(N+m+1) !}\left[\frac{(N+1)+m(N+1)}{N+1}\right] \\
=t^{N+m+1} \frac{N !}{(N+m+1) !}(m+1) .
\end{gathered}
$$

In conclusion, $c_{1, m}(t, N)$ is given by formula (72). Therefore,

$$
c_{1,1}(t, N)=2 t^{N+2} \frac{N !}{(N+2) !},
$$

OPTICS AND SPECTROSCOPY $\quad$ Vol. $103 \quad$ No. 22007 
$\partial_{t} c_{1,1}(t, N)=2 t^{N+1} \frac{N !}{(N+1) !}=c_{0,1}(t, N)+c_{1,0}(t, N)$.

Lemma 7. $c_{n, m}(t)$ has always the form

$$
C_{n, m}(t, N)=a_{n, m} \frac{N !}{(N+n+m) !} t^{N+n+m} .
$$

Proof. From (71), one obtains that

$$
\begin{gathered}
c_{n, m}(t, N)=\int_{0}^{t} c_{n-1, m}\left(t_{1}, N\right) d t_{1} \\
+\int_{0}^{t} c_{n, m-1}\left(t_{1}, N\right) d t_{1} .
\end{gathered}
$$

By applying again (71) to the second term of the righthand side of (75), we find that

$$
\begin{aligned}
& c_{n, m}(t, N)=\int_{0}^{t} c_{n-1, m}\left(t_{1}, N\right) d t_{1} \\
& +\int_{0}^{t} \int_{0}^{t_{1}} c_{n-1, m-1}\left(t_{2}, N\right) d t_{1} d t_{2} \\
& +\int_{0}^{t} \int_{0}^{t_{1}} c_{n, m-2}\left(t_{2}, N\right) d t_{1} d t_{2}=\int_{0}^{t} c_{n-1, m}\left(t_{1}, N\right) d t_{1} \\
& +\int_{0}^{t} \int_{0}^{t_{1}} c_{n-1, m-1}\left(t_{2}, N\right) d t_{1} d t_{2} \\
& +\int_{0}^{t} \int_{0}^{t_{1} t_{2}} c_{n-1, m-2}\left(t_{3}, N\right) d t_{1} d t_{2} d t_{3} \\
& +\iint_{0}^{t} \iint_{0}^{t_{1} t_{2}} c_{n, m-3}\left(t_{3}, N\right) d t_{1} d t_{2} d t_{3}=\ldots \\
& =\int_{0}^{t} \int_{0}^{t_{1}} \ldots \int_{0}^{t_{m-1}} c_{n, 0}\left(t_{m}\right) d t_{1} \ldots d t_{m} \\
& +\sum_{h=0}^{m-1} \iint_{0}^{t_{1}} \ldots \int_{0}^{t_{h-1}} c_{n-1, m-h}\left(t_{h+1}\right) d t_{1} \ldots d t_{h+1} .
\end{aligned}
$$

By (72), the first term of the right-hand side of (75) is equal to

$$
\begin{gathered}
\iint_{0}^{t t_{1}} \ldots \int_{0}^{t_{m-1}} \frac{n t_{m}^{N+n}}{(N+1)(N+2) \ldots(N+n)} d t_{1} \ldots d t_{m} \\
\quad=\frac{n t^{N+n+m}}{(N+1)(N+2) \ldots(N+n+m)}
\end{gathered}
$$

Moreover, the second term of the right-hand side of (75) depends only on $n-1$, therefore (75) gives an iteration formula to determine $\left\{c_{n, m}(t)\right\}_{n, m \in \mathbf{N}}$.

Lemma 8. The coefficients $\left(a_{m, n}\right)$ in (74) have the form

$$
a_{m, n}=\left(\begin{array}{c}
n+m \\
m
\end{array}\right)=\frac{(n+m) !}{n ! m !} .
$$

\section{Proof.}

$$
\begin{gathered}
a_{1,1}=a_{0,1}+a_{1,0}=2, \\
a_{2,1}=a_{1,1}+a_{2,0}=2+1=3, \\
a_{3,1}=a_{2,1}+a_{3,0}=3+1=4, \\
a_{n, 1}=n+1=\left(\begin{array}{c}
n+1 \\
1
\end{array}\right), \\
a_{n, 2}=a_{n-1,2}+a_{n, 1}=a_{n-1,2}+(n+1) \\
=a_{n-2,2}+m+(m+1)=a_{n-2,2 t} \sum_{k=n}^{n+1} k \\
=a_{n-3,2}+(n-1)+\sum_{k=n}^{n+1} k=a_{n-3}+\sum_{k=n-1}^{n+1} k \\
=\ldots=a_{n-\alpha, 2}+\sum_{n-\alpha+2}^{n+1} k, \\
\alpha=1,2, \ldots, n-1 ; \quad a_{n-(n-1), 2}=a_{1,2}=a_{2,1}=3, \\
a_{n, 2}=3+\sum_{k=3}^{n+1} k=\sum_{k=1}^{n+1} k \\
=1 / 2(n+1)(n+2)=\left(\begin{array}{c}
n+2 \\
2
\end{array}\right) .
\end{gathered}
$$

Now suppose that

$$
a_{n, \alpha}=\left(\begin{array}{c}
n+\alpha \\
\alpha
\end{array}\right)
$$


then

$$
\begin{gathered}
a_{n, \alpha+1}=a_{n-1, \alpha+1}+\left(\begin{array}{c}
n+\alpha \\
\alpha
\end{array}\right) \\
=a_{n-2, \alpha+1}+\left(\begin{array}{c}
n-1+\alpha \\
\alpha
\end{array}\right)+\left(\begin{array}{c}
n+\alpha \\
\alpha
\end{array}\right) \\
=a_{n-2, \alpha+1}+\sum_{k=n-1=(n-2)+1}^{n}\left(\begin{array}{c}
k+\alpha \\
\alpha
\end{array}\right) \\
=a_{n-3, \alpha+1}+\left(\begin{array}{c}
n-3+\alpha \\
\alpha
\end{array}\right)+\sum_{k=(n-2)+1}^{n}\left(\begin{array}{c}
k+\alpha \\
\alpha
\end{array}\right) \\
=a_{n-3, \alpha+1}+\sum_{k=(n-3)+1}^{n}\left(\begin{array}{c}
k+\alpha \\
\alpha
\end{array}\right) \\
=\ldots=a_{n-\beta, \alpha+1}+\sum_{k=(n-\beta)+1}^{n}\left(\begin{array}{c}
k+\alpha \\
\alpha
\end{array}\right) ;
\end{gathered}
$$

we stop at $n-\beta=1 \Leftrightarrow \beta=n-1$, which gives

$$
\begin{gathered}
a_{n, \alpha+1}=a_{1, \alpha+1}+\sum_{k=2}^{n}\left(\begin{array}{c}
k+\alpha \\
\alpha
\end{array}\right) \\
=\left(\begin{array}{c}
1+\alpha \\
\alpha
\end{array}\right)+\sum_{k=2}^{n}\left(\begin{array}{c}
k+\alpha \\
\alpha
\end{array}\right)=\sum_{k=1}^{n}\left(\begin{array}{c}
k+\alpha \\
\alpha
\end{array}\right) .
\end{gathered}
$$

So the induction is true if

$$
\sum_{k=1}^{n}\left(\begin{array}{c}
k+\alpha \\
\alpha
\end{array}\right)=\left(\begin{array}{c}
n+1+\alpha \\
\alpha+1
\end{array}\right) .
$$

By induction this is equivalent to

$$
\left(\begin{array}{c}
n+\alpha \\
\alpha+1
\end{array}\right)+\left(\begin{array}{c}
n+\alpha \\
\alpha
\end{array}\right)=\left(\begin{array}{c}
n+\alpha+1 \\
\alpha+1
\end{array}\right),
$$

which is identically satisfied. In fact the left-hand side of the identity

$$
\left(\begin{array}{c}
n \\
k+1
\end{array}\right)+\left(\begin{array}{l}
n \\
k
\end{array}\right)=\left(\begin{array}{l}
n+1 \\
k+1
\end{array}\right)
$$

is equal to

$$
\begin{gathered}
\frac{n !}{(k+1) !(n-k-1) !} \\
+\frac{n !}{k !(n-k) !} \frac{n !}{k !(n-k-1) !}\left[\frac{1}{k+1}+\frac{1}{n-k}\right] \\
=(\ldots)\left[\frac{n-k+k+1}{(k+1) n-k}\right]=(\ldots)\left[\frac{n+1}{(k+1) n-k}\right] \\
=\frac{(n+1)^{\prime}}{(k+1) !(n-k) !}=\left(\begin{array}{l}
n+1 \\
k+1
\end{array}\right) .
\end{gathered}
$$

In conclusion, (76) holds.
Combining Lemma 8 with (74) we obtain

$$
\begin{gathered}
c_{n, m}(t)=\left(\begin{array}{c}
n+m \\
m
\end{array}\right) \frac{N !}{(N+n+m) !} t^{N+n+m} \\
=\frac{(n+m) !}{n ! m !} \frac{N !}{(N+n+m) !} t^{N+n+m} \\
=\frac{1}{n ! m !}\left(\begin{array}{c}
N+n+m \\
n+m
\end{array}\right)^{-1} t^{N+n+m} .
\end{gathered}
$$

Therefore, using the notation:

$$
\begin{gathered}
\Phi(h, j, n, m) \\
:=X_{+}^{h-1}(-i \mathbf{p})^{+}\left(F_{g} \mid X_{+}^{n-h} T_{G} X_{-}^{j-1} F_{g}\right)(-i \mathbf{p}) X_{-}^{m-j},
\end{gathered}
$$

we find

$$
\begin{gathered}
V\left(t, T_{G}\right)=\sum_{n, m=11 \leq h \leq n, 1 \leq j \leq m}^{\infty} \Phi(h, j, n, m) \\
\times \frac{c_{h-1, j-1}(t, n+m+1-h-j)}{(n-h) !(m-j) !(n+m+1-h-j)} \\
=\sum_{n, m} \sum_{h, j} \Phi(h, j, n, m) \\
\times \frac{1}{(n-h) !(m-j) !(n+m+1-h-j)} \\
\times \frac{1}{(h-1) !(j-1) !\left(\begin{array}{c}
n+m-1 \\
h+1
\end{array} t^{n+m+1-h-j+h-1+j-1}\right.} \\
=\sum_{n, m} \sum_{h, j}(h, j, n, m)\left(\begin{array}{c}
n+m-1 \\
h+j-2
\end{array}\right)^{-1}\left(\begin{array}{c}
n+m-h-j \\
n-h
\end{array}\right) \\
\times\left(\begin{array}{c}
h+j-2 \\
h-1
\end{array}\right) \frac{1}{(h+j-2) !} t^{n+m-1}
\end{gathered}
$$

and from the identity

$$
\begin{gathered}
\frac{(h+j-2) !(n+m+1-h-j) !}{(n+m-1) !} \\
\times\left(\begin{array}{c}
h+j-2 \\
h-1
\end{array}\right) \frac{1}{(n+m-h-j+1) !(h+j-2) !} \\
=\frac{1}{(n+m-1) !}
\end{gathered}
$$

we finally obtain

$$
\begin{gathered}
V\left(t, T_{G}\right)=\sum_{m, n=1}^{\infty} \sum_{h=1}^{n} \sum_{j=1}^{m} X_{+}^{h-1}(-i \mathbf{p})^{+} \\
\times\left(F_{g} \mid X_{+}^{n-h} T_{G} X_{-}^{j-1} F_{g}\right)(-i \mathbf{p}) X_{-}^{m-j} \\
\times \frac{t^{n+m-1} 1}{(n+m-1) !}\left(\begin{array}{c}
n+m-h-j \\
n-h
\end{array}\right)\left(\begin{array}{c}
h+j-2 \\
h-1
\end{array}\right) .
\end{gathered}
$$

OPTICS AND SPECTROSCOPY Vol. 103 No. $2 \quad 2007$ 


\section{REFERENCES}

1. L. Accardi, Y. G. Lu, and I. Volovich, Quantum Theory and Its Stochastic Limit (Springer, New York, 2002).

2. H. M. Srivastava and P. W. Karlsson, Multiple Gaussian Hypergeometric Series (Wiley, New York, 1985).

3. L. Accardi, and Y. G. Lu., in Proceedings of the International Workshop on Mathematical Physics, Siena, Italy, 1991, Ed. by F. Guerra and M. Loffredo (World Scientific, 1992), pp. 16-29; Volterra Preprint No. 89 (1992).
4. L. Accardi and Y. G. Lu. Commun. Math. Phys. 180, 605 (1996); Volterra Preprint No. 26 (1992).

5. L. Accardi, S. V. Kozyre, and I. V. Volovich J. Phys. Math. Gen. 32, 3485 (199); http://xxx.lanl.gov/abs/q$\mathrm{alg} / 9807137$.

6. J. Gough, Volterra Preprint (1996).

7. J. Gough, Volterra Preprint No. 244 (1996).

8. Y. G. Lu, Random Oper. Stoch. Equ. 4 (1) 93 (1996). 